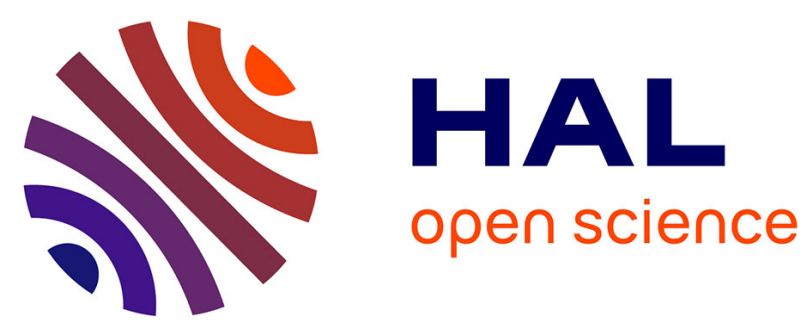

\title{
Intérêts comparés de l'indexage biologique sur semis de pêcher GF 305 et du diagnostic immunoenzymatique ELISA pour la détection de virus des espèces fruitières
}

Pascale Pracros, Gaston Detienne, Chantai Sarraquigne, Jean Dunez

\section{- To cite this version:}

Pascale Pracros, Gaston Detienne, Chantai Sarraquigne, Jean Dunez. Intérêts comparés de l'indexage biologique sur semis de pêcher GF 305 et du diagnostic immunoenzymatique ELISA pour la détection de virus des espèces fruitières. Agronomie, 1981, 1 (7), pp.535-540. hal-00884292

\section{HAL Id: hal-00884292 \\ https://hal.science/hal-00884292}

Submitted on 1 Jan 1981

HAL is a multi-disciplinary open access archive for the deposit and dissemination of scientific research documents, whether they are published or not. The documents may come from teaching and research institutions in France or abroad, or from public or private research centers.
L'archive ouverte pluridisciplinaire $\mathbf{H A L}$, est destinée au dépôt et à la diffusion de documents scientifiques de niveau recherche, publiés ou non, émanant des établissements d'enseignement et de recherche français ou étrangers, des laboratoires publics ou privés. 


\title{
Intérêts comparés de l'indexage biologique sur semis de pêcher GF 305 et du diagnostic immunoenzymatique ELISA pour la détection de virus des espèces fruitières
}

\author{
Pascale PRACROS $\left({ }^{1}\right)$, Gaston DETIENNE $\left({ }^{*}\right)$, Chantal SARRAQUIGNE \& Jean DUNEZ $\left({ }^{*}\right)$ \\ Service de la Protection des Végétaux, F 33150 Cenon
}

${ }^{*}$ ) I.N.R.A., Station de Pathologie végétale, Centre de Recherches de Bordeaux, F 33140 Pont de la Maye.

\author{
RÉSUMÉ \\ Arbres fruitiers, \\ Virus, \\ Virus des taches chloroti- \\ ques du pommier, \\ Virus des taches \\ annulaires nécrotiques \\ des Prunus, \\ Virus du nanisme \\ du prunier, \\ Indexage biologique, \\ Indexage sérologique, \\ Techniques \\ immunoenzymatiques, \\ ELISA.
}

\begin{abstract}
La lignée de pêcher GF 305 est très largement utilisée pour l'indexage en serre des virus des espèces fruitières: elle est bien connue pour sa sensibilité et sa polyvalence. La comparaison de la méthode d'indexage sur cet indicateur et de la technique ELISA a été réalisée à partir de 534 échantillons prélevés sur différentes espèces fruitières à noyau. Le nombre total d'échantillons reconnus infectés est supérieur par utilisation du test ELISA que par l'emploi de l'indexage sur GF 305. CLSV et virus du groupe des ILAR virus sont mis en évidence respectivement sur 68 et 54 échantillons qui n'induisent pas de réaction sur GF 305. Cependant la situation inverse se rencontre également, notamment dans la recherche du CLSV; 64 échantillons négatifs en ELISA induisent une réaction sur GF 305. Il n'est pas impossible, dans ce cas, qu'il existe certaines confusions avec des symptômes dus aux virus du groupe ILAR, à certaines souches de CLSV ou à des maladies d'origine inconnue. Bien que certaines difficultés associées à la présence de souches particulières demeurent, le test ELISA apparaît dès maintenant globalement supérieur à l'indexage sur GF 305 dans le contrôle de routine de différents virus des espèces fruitières et notamment dans l'étude des réinfections naturelles. Néanmoins, la lignée de pêcher GF 305 garde son intérêt par son aptitude à déceler des maladies non encore justiciables de diagnostic immunologique.
\end{abstract}

\section{SUMMARY}

Fruit trees,

Viruses,

Apple chlorotic leaf spot virus,

Prunus necrotic ring spot virus,

Prune dwarf virus, Bio-assay, Serological indexing, Immunoenzymatic techniques, ELISA.

\begin{abstract}
Bioassay on peach seedlings GF 305 and immunoenzymatic assay (ELISA) for the detection of fruit tree viruses

The peach seedling GF 305 is an indicator plant commonly used in the greenhouse for detecting several fruit tree viruses; it is well known for its sensitivity and its potentiality to detect many virus and virus-like diseases. Comparative indexing of 534 stone fruit tree samples has been carried out on GF 305 and by ELISA for the detection of apple chlorotic leaf spot (CLSV) and two viruses of the ILAR virus group, Prunus necrotic ring spot (NRSV) and prune dwarf (PDV). Number of infected samples was higher in ELISA than with GF 305 (276/238). Nevertheless the correlation was not perfect. The ELISA technique seems to be more reliable: CLSV and viruses of the ILAR virus group are identified by ELISA in respectively, 68 and 54 samples which do not induce any symptom on GF 305 . But conversely, in the search for CLSV, 64 samples which induce symptoms on GF 305 do not give any reaction in ELISA : this could mean some confusion of symptoms of the GF 305 or reflect the existence of particular strains. In another work we demonstrate that most known NRSV or PDV strains can be detected by ELISA in the conditions used for the present investigations. Apple mosaic virus, a virus that we have frequently detected in stone fruit trees, especially in almond trees, was not investigated with ELISA and could be responsible for symptom expression on GF 305.

This paper shows that neither GF 305 nor ELISA are able to detect all the cases of infection but ELISA appears clearly superior to the GF 305 bioassay in a routine indexing scheme, especially for recontamination investigations.
\end{abstract}

\section{INTRODUCTION}

L'indexage des maladies à virus des espèces fruitières a été, pendant de longues années, réalisé par inoculation de

(1) Adresse actuelle: I.N.R.A., Station de Zoologie agricole, F 33140 Pont de la Maye. variétés ou espèces indicatrices sensibles cultivées en pépinière ou en serre. Les semis de pêcher sont connus pour leur bonne sensibilité et une polyvalence assez grande; par ailleurs, leur possibilité de production en serre et leur relative rapidité de réponse permettent de détecter de nombreuses maladies en quelques semaines et en font un 
type d'indicateur très largement utilisé. Parmi les différents semis de pêcher disponibles, la lignée homozygote GF 305 a, outre l'intérêt des autres semis, celui d'avoir une croissance très homogène : l'étude comparée de la croissance des plantes infectées et de celle de plantes non infectées fournit un critère supplémentaire d'infection (BERNHARD \& MARÉNAUD, 1963). Parmi les maladies à virus, dont l'agent causal a été bien identifié et est décelable sur le.semis de pêcher GF 305 figurent les virus du groupe des ILAR virus (Prunus necrotic ring spot virus - NRSV - prune dwarf virus PDV - et apple mosaic virus - AMV), le virus des taches foliaires du pommier (apple chlorotic leaf spot virus CLSV), le virus de la sharka (plum pox virus - PPV). Ces différents virus ont été isolés, identifiés, purifiés et sont donc depuis plusieurs années justiciables d'un indexage sérologique. L'utilisation d'un tel indexage a été longtemps limitée du fait du manque de sensibilité de certaines techniques immunologiques, double diffusion en milieu gélosé (RICHTER \& KLEINHEMPEL, 1967), immunodiffusion radiale (CASPER, 1975). Les conditions du diagnostic ont été complètement modifiées par l'introduction des techniques immunoenzymatiques dont la sensibilité est au moins égale à celle obtenue par l'utilisation de plantes indicatrices (Clark \& AdAMS, 1977 ; LISTER, 1978 ; BAR JOSEPH et al., 1979). Il était intéressant, en se plaçant dans les conditions d'un contrôle de routine, de comparer les résultats obtenus par le test immunoenzymatique ELISA et l'indexage sur GF 305. Nous présentons ici les résultats de l'analyse par ces 2 méthodes de plus de 500 échantillons originaires de différentes espèces fruitières et ornementales appartenant au genre Prunus. Les virus les plus fréquents chez ces espèces (CLSV, PDV et NRSV) y ont été recherchés.

\section{MATÉRIEL ET MÉTHODES}

\section{A. Matériel}

\section{Immunsérums}

Les sérums anti-PDV (PV AS 33) et anti-NRSV (PV AS 22) ont été acquis auprès de l'American Type Culture Collection. Le sérum anti-CLSV (souche P 863) a été préparé au laboratoire (DETIENNE et al., 1981).

Les titres de ces sérums déterminés par double diffusion en milieu gélosé sont respectivement de $1: 2048,1: 512$, $1: 1024$ pour PDV, NRSV et CLSV.

\section{Immunoglobulines}

Les IgG ont été purifiées par chromatographie d'affinité sur Sépharose-protéine A (Pharmacia). Le couplage avec la phosphatase alcaline (Sigma type VII) a été réalisé en présence de glutaraldéhyde à 0,06 p. 100 .

\section{Souches de virus}

Les souches de virus utilisées comme « témoins " dans les contrôles étaient NRSV-G (Fulton) PDV-B (Fulton) et CLSV-P 863.

\section{Plantes indicatrices}

L'indexage biologique est effectué en serre sur semis de pêcher GF 305. Cinq répétitions sont réalisées par échantillon indexé. Les notations sont réalisées un mois après l'inoculation puis les plantes sont rabattues et une $2^{\mathrm{e}}$ notation est pratiquée 4 à 6 semaines plus tard.

\section{B. Méthodes}

Le test ELISA est réalisé dans des plaques de microtitration Dynatech M129 B selon la technique "sandwich» (Voller et al., 1976; ClARK \& AdAMS, 1977).

Les IgG sont utilisées à une concentration de $5 \mu \mathrm{g} / \mathrm{ml}$ : les conjugués sont dilués 1:1000 (NRSV, PDV) et 1:500 (CLSV) et le substrat employé à la concentration de $1 \mathrm{mg} / \mathrm{ml}$.

Les échantillons sont préparés par broyage de feuilles dans une presse électrique à rouleaux E. Polhäne en présence d'une solution tampon constituée de PBS-Tween-PVP (CLARK \& ADAMS, 1977) additionnée de 0,005 $\mathrm{M} \mathrm{MgCl}_{2}$, 0,2 p. 100 diaminodipropylamine et 2,5 p. 100 nicotine. Cette solution, bien adaptée à la détection du CLSV (Detienne et al., 1981) chez les différentes espèces fruitières convient parfaitement pour NRSV et PDV. Le rapport poids de feuilles (en g) : volume du milieu d'extraction $(\mathrm{ml})$ est compris entre $1: 5$ et $1: 10$. Deux puits de la plaque de microtitration sont utilisés par échantillon et 12 réservoirs par plaque sont réservés aux extraits sains témoins.

Les lectures sont réalisées après, en général, $1 \mathrm{~h}$ d'incubation du substrat à la température du laboratoire grâce à un lecteur de plaques Biomérieux LOV 1. Les D.O. des témoins sains ne sont pas mentionnées: elles sont au maximum de 0,05 unité de D.O. au-dessus du blanc (milieu d'extraction). Les densités optiques présentées par les tableaux ont été diminuées des D.O. obtenues avec les échantillons témoins sains.

Les semis de pêcher GF 305 sont inoculés par la technique des implants tissulaires (chip budding). Les écorces utilisées ici et les feuilles employées pour le test ELISA sont prélevées sur le même rameau.

\section{RÉSULTATS}

\section{A. Résultats comparés de l'indexage sur GF 305 et du test ELISA}

Le tableau 1 regroupe les résultats globaux de l'indexage de 534 échantillons. Il faut d'abord signaler que ces indexages ont montré un taux d'infection par le PDV extrêmement faible $(2,5$ p. 100$)$ et que les résultats pour les virus du

\section{TABLEAU 1}

Résultats comparés de l'analyse de 534 échantillons végétaux par indexage sur le semis de pêcher GF305 et par le test ELISA (recherche du CLSV, NRSV et PDV)

Comparative results of indexing of 534 samples by indexing onto GF 305 and by the ELISA test (search for CLSV, NRSV and PDV)

\begin{tabular}{lcc}
\hline & \multicolumn{2}{c}{ Technique d'indexage } \\
& GF 305 & ELISA \\
\hline $\begin{array}{l}\text { Nombre total d'échantillons infectés } \\
\text { Nombre total d'échantillons non }\end{array}$ & 238 & 276 \\
$\begin{array}{l}\text { infectés } \\
\text { Nombre total d'échantillons douteux }\end{array}$ & 293 & 253 \\
$\begin{array}{l}\text { Nombre d'échantillons infectés par lc } \\
\text { CLSV }\end{array}$ & 218 & 5 \\
$\begin{array}{l}\text { Nombre d'échantillons infectés par le } \\
\text { NRSV ou PDV }\end{array}$ & 127 & $167\left({ }^{2}\right)$ \\
\end{tabular}

(1) 75 p. 100 des résultats concordants entre GF 305 ct ELISA.

(2) 87 p. 100 des résultats concordants entre GF 305 et ELISA. 
groupe des ILAR virus prennent donc essenticllement en considération le NRSV.

La $1^{\text {re }}$ remarque est que la technique ELISA détecte un nombre de plantes infectées supérieur au nombre de plantes induisant des réactions sur GF 305 (276/238). Cette observation est également valable pour chaque type de virus, aussi bien pour le CLSV (223/218) que pour les virus du groupe ILAR virus (167/127).

La $2^{\mathrm{e}}$ remarque est que le taux de corrélation entre les résultats obtenus par chacune des techniques est assez nettement inférieur à 100 . Les résultats sont identiques seulement pour 75 p. 100 des échantillons analysés, dans le cas du CLSV, et 87 p. 100 , dans le cas des virus du groupe des ILAR virus.

Parmi les situations de désaccord entre ELISA et GF 305, existent d'abord des cas limites souvent difficiles à interpréter avec des valeurs insuffisantes pour être parfaitement significatives en ELISA et des réactions très faibles du GF 305. Par ailleurs, on note des cas de désaccord flagrant avec de forts symptômes sur GF 305 et pas de réaction en ELISA ou vice versa. Le tableau 2 illustre, par quelques exemples, de telles situations.

Nous avons recherché l'origine de ces différences et la $1^{\text {rc }}$ hypothèse réside dans la diversité possible des souches. La technique ELISA est, en effet, connue pour présenter une spécificité de «souches» plus étroite que d'autres techniques immunologiques. Dans le cas du CLSV, nous avons démontré (DETIENNE et al., 1981) que les variations antigéniques entre les souches connues étaient faibles et que toutes les souches connues à ce jour pouvaient être décelées. La technique ELISA apparaissait à cet égard plus polyvalente que l'indexage sur GF 305 : il est donc tout à

TABLEAU 2

Quelques exemples de désaccord entre les résultats du test GF 305 et le test ELISA

Some exemples of discrepancy between GF 305 and ELISA results

Référence et nature de l'échantillon $\begin{gathered}\text { Technique d'indexage } \\ \text { GF } 305\left({ }^{1}\right) \quad \text { ELISA }\left({ }^{2}\right)\end{gathered}$

Tableau 2 A : Recherche du NRSV

Indexing of NRSV

$54 \mathrm{JP} 11$ ( $P$. pissardii)

$18 \mathrm{~W} 30$ ( $P$. armeniaca)

57 M $8(P$. domestica $)$

45 JP 39 ( $P$. domestica $)$

$18 \mathrm{~W} 40$ ( $P$. domestica)

$37 \mathrm{~S} 12(P$. cerasifera $)$

\begin{tabular}{ll}
- & 0,863 \\
- & 0,600 \\
- & 0,633 \\
++ & 0,024 \\
+ & 0,033 \\
+ & 0,015 \\
\hline
\end{tabular}

Tableau 2 B : Recherche du CLSV

Indexing of CLSV

47 A 133 (P. domestica)

47 A $100(P$. domestica $)$

47 A $24(P$. domestica $)$

45 JP 6 ( $P$. pissardii)

33 P 1 ( $P$. domestica)

87 LJC 4 ( $P$. cerasifera $)$

$\begin{array}{ll}- & 1,294 \\ - & 0,610 \\ - & 1,506 \\ ++ & 0,029 \\ ++ & 0,022 \\ ++ & 0,028\end{array}$

(1) le nombre de croix reflète l'intensité des symptômes.

(2) valeurs de la D.O. à $405 \mathrm{~nm}$.

fait logique, dans les essais rapportés ici, que le nombre d'échantillons reconnus infectés par le CLSV soit plus grand par la technique ELISA que par indexage sur GF 305. Des résultats obtenus par ailleurs avec le groupe des ILAR virus montrent que la plupart des souches connues peuvent également être décelées par la technique ELISA dans les conditions de nos essais.

Le pêcher GF 305, également connu pour sa polyvalence (BERNHARD et al., 1969), réagit au CLSV et aux virus du groupe ILAR par 2 types différents de réactions. Dans le $1^{\text {er }}$ cas, il extériorise le symptôme connu sous le nom de «dark green sunken mottle»; dans le $2^{c}$, les plantes présentent des marbrures, des nécroses plus ou moins fortes et une croissance souvent réduite. Il n'est pas impossible que certains désaccords soient dus à des confusions de symptômes. Par exemple, dans la recherche du CLSV, 64 échantillons sont positifs sur GF 305 et négatifs en ELISA. Or, parmi ces 64 échantillons, dont 16 seulement provoquent sur GF 305 des symptômes nets de CLSV, une forte proportion apparaît infectée par le NRSV tant par le test ELISA (36) que sur GF 305 (34) : on ne peut écarter totalement l'hypothèse selon laquelle la réaction du GF 305 associée, dans les notations, au CLSV ait été provoquée par une souche faible de NRSV. Par ailleurs, la présence fréquente d'infections mixtes rend parfois difficiles la lecture et l'interprétation du test et il faut garder à l'esprit que le GF 305 peut extérioriser une réaction à une infection par d'autres virus que ceux qui ont été recherchés ici ou par différentes maladies transmissibles d'origine inconnue.

Il faut enfin signaler que, dans le cas de résultats concordants, il n'existe pas de corrélation entre la sévérité des symptômes sur GF 305 et l'intensité de la réaction en ELISA (tabl. 3), ces différences reflétant à la fois des différences de concentration du virus dans l'inoculum et la diversité des souches de virus rencontrées.

\section{TABLEAU 3}

Quelques exemples comparés des résultats d'indexage sur GF 305 et du test ELISA

Comparative indexing of some samples onto GF 305 and by ELISA

Référence et nature de l'échantillon Technique d'indexage GF $305\left(^{1}\right) \quad \operatorname{ELISA}\left({ }^{2}\right)$

Tableau 3 A : Recherche de virus du groupe des ILAR virus Indexing of viruses of the ILAR virus group

\begin{tabular}{|c|c|c|}
\hline 45 JP 6 ( $P$. pissardii $)$ & ++ & 0,183 (NRSV) \\
\hline 45 JP 33 ( $P$. domestica) & ++ & 0,151 (NRSV) \\
\hline $37 \mathrm{~S} 19$ ( $P$. domestica $)$ & + & 0,975 (PDV) \\
\hline 54 M 2 (P. domestica) & +++ & 0,283 (PDV) \\
\hline $41 \mathrm{DL} 2$ ( $P$. pissardii) & + & $>2 \quad(\mathrm{PDV})$ \\
\hline
\end{tabular}

Tableau 3 B : Recherche du CLSV

Indexing of CLSV

$45 \mathrm{JP} 14$ ( $P$. pissardii)

$18 \mathrm{DW} 36$ ( $P$. domestica $)$

$54 \mathrm{M} 2(P$. domestica $)$

33 P 15 ( $P$. domestica)

41 DL $2(P$. pissardii)

$\begin{array}{cc}++ & 0,100 \\ + & 1,257 \\ ++ & 0,238 \\ +++ & 0,244 \\ + & 1,007\end{array}$

(1) le nombre de croix reflète l'intensité des symptômes.

${ }^{(2)}$ valeurs de la D.O. à $405 \mathrm{~nm}$. 


\section{B. Sensibilité du test ELISA}

Si, dès maintenant, le test ELISA apparaît capable de détecter plus de contaminations que l'indexage sur GF 305 , il est vraisemblable que de légères modifications dans sa mise en ouvre permettront d'augmenter encore la sensibilité de la technique immunoenzymatique. Le seuil habituellement utilisé au laboratoire pour noter une réaction positive est de 0,1 unité de D.O. au-dessus de la valeur de l'échantillon témoin après $1 \mathrm{~h}$ d'incubation du substrat. Ce seuil, supérieur à certaines valeurs utilisées dans la littérature, a été choisi pour fournir un maximum de garanties à l'interprétation de résultats positifs. Plutôt que de rechercher l'utilisation, dans les mêmes conditions, d'un seuil plus bas dont l'interprétation serait difficile, compte tenu de légères variations à l'intérieur de la plaque de microtitration, il nous a semblé préférable d'augmenter la sensibilité du test par un allongement du temps d'hydrolyse du substrat. La figure 1 présente les résultats notés après différents temps d'incubation du substrat lors de la recherche du CLSV dans 4 échantillons végétaux infectés comparés à un échantillon témoin sain et à une préparation purifiée de CLSV à $1 \mathrm{ng}$ de virus par $\mathrm{ml}$. L'évolution des D.O. à la température du laboratoire montre d'abord que, dans le cas de systèmes IgG-conjugué ne présentant pas de réactions non spécifiques, les valeurs du témoin restent faibles. Après 30 min d'incubation, 3 échantillons et la préparation purifiée de CLSV présentent des valeurs non significatives; après $2 \mathrm{~h}$ d'incubation les chiffres obtenus sont devenus significatifs et le sont encore plus après $24 \mathrm{~h}$.
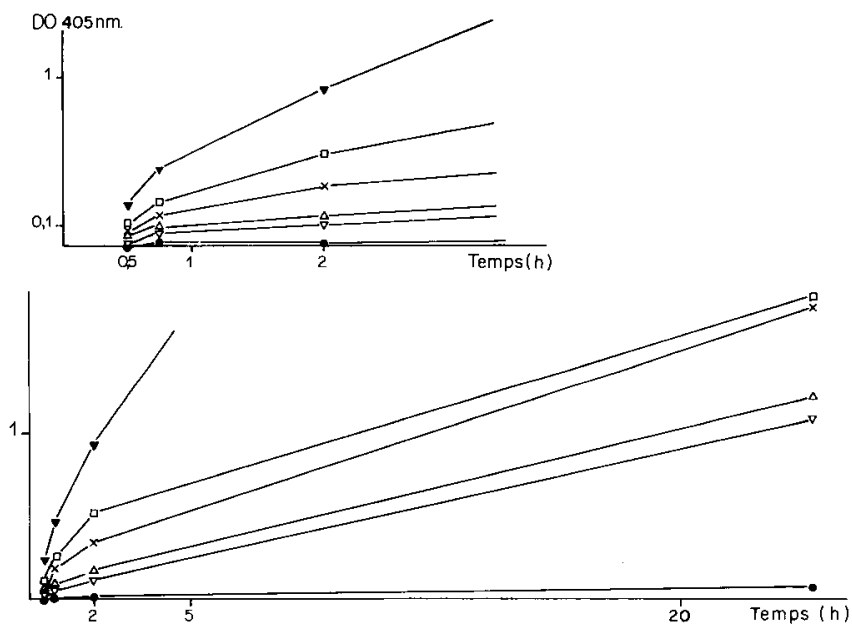

Figure 1

Sensibilité de la technique ELISA en relation avec le temps d'hydrolyse du substrat (incubation à la température du laboratoire)

- Echantillon sain,

$\nabla$ Préparation purifiée de CLSV à $1 \mathrm{ng} / \mathrm{ml}$,

$\triangle$ Echantillon infecté $n^{\circ} 10-72$,

$\nabla$ Echantillon infecté $n^{\circ} 10-73$,

$\square$ Echantillon infecté $n^{\circ} 10-75$

$\times$ Echantillon infecté $n^{\circ} 10-78$.

Variation of the sensitivity of the ELISA technique according to the duration the incubation of substrate (at room temperature).

- healthy sample,

$\nabla$ purified CLSV suspension $(1 \mathrm{ng} / \mathrm{ml})$

$\triangle$ infected sample $n^{\circ} 10-72$,

$\boldsymbol{\nabla}$ infected sample $n^{\circ} 10-73$,

$\square$ infected sample $n^{\circ} 10-75$,

$\times$ infected sample $n^{\circ} 10-78$.
Un même résultat peut être obtenu simplement et plus rapidement par une incubation du substrat à une température de $37^{\circ} \mathrm{C}$.

\section{CONCLUSIONS}

Ces résultats ne permettent pas encore une évaluation de l'intérêt du test ELISA généralisée à l'ensemble des espèces fruitières; en effet les nombres d'échantillons par espèce étaient très différents : sur les 534 échantillons analysés, 162 étaient constitués de porte-greffes appartenant à différentes espèces de Prunus ( $P$. mariana, $P$. domestica, $P$. cerasifera) et 267 étaient issus de variétés commerciales de prunier notamment de prunier d'Ente. Pour les espèces $P$. persica (pêcher), $P$. armeniaca (abricotier) et $P$. pissardii seulement 29,14 et 52 échantillons respectivement ont été analysés. Aucun essai n'a été effectué pour l'amandier mais, dans des indexages réalisés dans le cadre d'un essai en collaboration avec la station italienne de Catane, le test ELISA s'est montré très performant avec cette espèce. Aucune espèce à pépins n'a été étudiée mais nous avons déjà souligné pour le CLSV qu'il n'existait aucune difficulté particulière à l'application du test ELISA au pommier ou au poirier (DETIENNE et al., 1981). Il serait, par ailleurs, ainsi que nous l'avons déjà indiqué, intéressant d'inclure la recherche de l'AMV par le test ELISA. Ce virus est, en effet, présent à la fois chez les espèces à pépins et les espèces à noyau et a été détecté fréquemment dans les contrôles d'amandiers que nous venons de mentionner. L'application du test ELISA à la recherche des virus du groupe des ILAR virus, utilisant des immunoglobulines NRSV, PDV et AMV, paraît être de nature à déceler la plupart des souches de ces virus : ces immunoglobulines détectent ainsi, respectivement, tulare mosaic, chlorotic necrotic ring spot et rose mosaic (FULTON, 1967 ; DETIENNE et al., en préparation).

Une des conclusions à tirer de ces investigations est la confirmation qu'aucune méthode d'indexage n'est réellement parfaite. Aussi sensible et polyvalent que soit le semis de pêcher GF 305, il ne détecte pas tous les cas d'infection et il en détecte moins que le test ELISA.

Ce test immunoenzymatique, dont la sensibilité peut encore être améliorée et qui apparaît dès lors plus intéressant que le semis de pêcher pour sa rapidité et ses possibilités d'automatisation, est un outil parfaitement bien adapté aux contrôles de routine du CLSV et des virus du groupe des ILAR virus, comme cela avait déjà été montré pour la sharka (ADAMS, 1978). Cependant, il ne remplacera pas totalement le GF 305 ni les autres indicateurs dans la production des plants de base indemnes de virus pour lesquels il faut rechercher le maximum de garanties. Sa simplicité de mise en œuvre, sa sensibilité et son coût peu élevé permettront de l'utiliser en $1^{\text {re }}$ analyse ; les plants qui n'auront pas réagi au test ELISA seront alors indexés sur GF 305 et les indicateurs habituels qui garderont tout leur intérêt pour la détection de souches particulières ou de maladies transmissibles non encore justiciables des techniques immunologiques. Le test ELISA sera extrêmement intéressant pour l'étude des réinfections en verger ou en pépinière comme l'a montré ADAMS (1978) pour la sharka et dans les échanges internationaux chaque fois qu'une analyse rapide sera nécessaire. 


\section{RÉFÉRENCES BIBLIOGRAPHIQUES}

Adams A. N., 1978. The detection of plum pox virus in Prunus species by enzyme linked immunosorbent assay (ELISA). Ann. appl. Biol., 90, 215-221.

Bar Joseph M., Gansey S. M., Gonsalves D., Moscovits M., Purcifull D. E., Clark M. F., Lobenstein G., 1979. The use of enzyme linked immunosorbent assay for detection of citrus tristeza virus. Phytopathology, 69, 190-194.

Bernhard R., Marénaud C., 1963. Une méthode plus sensible d'indexage sur pêcher : utilisation d'un index sélection, étude des variations de sa courbe de croissance. Phytopathol. méditerr., II (3) 209-219.

Bernhard R., Marénaud C., Sutić D., 1969. Le pêcher GF 305, indicateur polyvalent des virus des espèces à noyau. Ann. Phytopathol., 1 (4), 603-617.

Casper R., 1975. Serodiagnosis of plum pox virus. Acta horticult., 44, 171-172.

Clark M. F., Adams A. N., 1977. Characteristics of the microplate method of enzyme linked immunosorbent assay for the detection of plant viruses. J. gen. virol., 34, 475-483.
Clarke R. G., Converse R. H., Kosima M., 1980. Enzyme linked immunosorbent assay to detect potato leaf roll virus in potato tubers and viruliferous aphids. Plant Dis., 64, 43-45.

Detienne G., Delbos R., Dunez J., 1981. Use and versatility of the immunoenzymatic ELISA procedure in the detection of different strains of apple chlorotic leaf spot. Acta phytopathol. (sous presse).

Fulton R. W., 1967. Relationships among ring spot viruses of Prunus. Tagungsberichte 97, 123-138.

Lister R. M., 1978. Application of the enzyme-linked immunosorbent assay for detecting viruses in seeds and plants. Phytopatho$\log y, 68,1398-4000$.

Richter J., Kleinhempel H., 1967. Serologischer Nachweis von NEPO Virus in Obstgewnächsen. Tagungsberichte 97, 277-282.

Voller A., Bidwell D. E., Bartlett A., Clark M. F., Adams A. N., 1976. The detection of viruses by enzyme linked immunosorbent assay. J. gen. virol. 33, 165-167. 